\title{
UMA ANÁLISE DAS CONSEQUÊNCIAS DA IMPLANTAÇÃO DO CAMPUS DA UNIVERSIDADE FEDERAL DE UBERLÂNDIA NA EVOLUÇÃO URBANA DE MONTE CARMELO POR MEIO DE IMAGENS MULTIESPECTRAIS DE BAIXO CUSTO
}

\author{
Alexandre Fleury de Siqueira e Gomes \\ Universidade Federal de Uberlândia (UFU), Curso de Engenharia de Agrimensura e Cartográfica, Monte \\ Carmelo, MG, Brasil \\ alexandrefleury.sg@hotmail.com \\ George Deroco Martins \\ Universidade Federal de Uberlândia (UFU), Faculdade de Engenharia Civil, Monte Carmelo, MG, Brasil \\ deroco@ufu.br \\ João Vitor Meza Bravo \\ Universidade Federal de Uberlândia (UFU), Instituto de Geografia, Monte Carmelo, MG, Brasil \\ jvmbravo@gmail.com
}

\begin{abstract}
RESUMO
Este estudo analisa a evolução urbana de Monte Carmelo (MG), enquanto uma cidade de pequeno porte. A hipótese levantada neste artigo vincula a evolução da paisagem urbana carmelitana à instalação do campus da Universidade Federal de Uberlândia na cidade. O campus serviria como vetor de atração à expansão urbana e, ao mesmo tempo, agente catalisador de processos urbanos em toda a cidade. Selecionou-se Monte Carmelo como estudo de caso, pois essa é uma cidade pequena que sofre com a falta de dados espaciais: situação que impacta negativamente a construção de políticas efetivas de gestão territorial. Para cum prir com o objetivo, nesta pesquisa utilizou-se de imagens orbitais de baixo cu sto para estudar a dinâmica de evolução urbana carmelitana. Com essa proposta concretizada construiu-se um modelo alternativo para observação de uma cidade pequena, com tecnologia acessível aos gestores, preenchendo a lacuna de acessibilidade tecnológica. Por meio do processamento digital de imagens e de uma análise visual, foi possível quantificar e qualificar a evolução urbana local, entre os anos de 2013 e 2018 . Concluiu-se que o adensamento urbano de Monte Carmelo pode ser explicado pela chegada da Universidade Federal de Uberlândia à cidade, especialmente com o início das atividades de ensino em 2011.
\end{abstract}

Palavras-chave: Cidade pequena. Adensamento urbano. Expansão de Fronteira. Geoinformação. Universidades.

\section{AN ANALYSIS INTO THE CONSEQUENCES BROUGHT BY THE ARRIVAL OF THE FEDERAL UNIVERSITY OF UBERLANDIA CAMPUS ON THE URBAN EVOLUTION OF MONTE CARMELO BY MEANS OF LOW COST MULTISPECTRAL IMAGES}

\begin{abstract}
In this paper we have analyzed the urban evolution of Monte Carmelo, which is a sm all city of Minas Gerais, Brazil. Here the hypothesis links the urban landscape evolution of Monte Carmelo to the installation of the Federal University of Uberlândia campus in the city. Therefore, we have argued that the campus has served as a vector of attraction for the urban expansion and, at the same time, promoted changes in the urban processes for other regions of Monte Carmelo. Thereby, we have also selected Monte Carmelo as a case study, because it is a small city that suffers with the lack of spatial data, a situation that generates negative impacts on the urban management. Therefore, we have used low cost satellite images to analyze the urban evolution dynamics of Monte Carmelo. Consequently, we have filled a gap in technological accessibility, by adapting a method that aids city managers to observe a small urban system with low cost geoinformation. Through DIP and a visual analysis approach, we have quantified/qualified the urban evolution of Monte Carmelo throughout the years 2013-2018. In doing so, we have concluded that the urban densification could be explained by the Federal University of Uberlândia cam pus arrival.
\end{abstract}

Keywords: Small city. Urban densification. Frontier expansion. Geoinformation. Universities. 


\section{INTRODUÇÃO}

O crescimento acelerado da população mundial fez aumentar o número de aglomerados urbanos (HARVEY, 2000; SANTOS, 2012; MARSHALL, 2011; BATTY e MARSHALL, 2011; BUHAUG e URDAL, 2013). Nesse cenário, as pesquisas científicas têm dado atenção especial aos grandes sistemas urbanos, considerando suas relações naturalmente complexas, bem como, por capitanearem grande parte da população mundial (HARVEY, 2000; MULLIGAN e CRAMPTON, 2005; BELL e JAYNE, 2009; KANAI; GRANT; JIANU, 2017; VARNA; ADAMS; DOCHERTY, 2020).

Nos últimos anos, as cidades pequenas ganharam notoriedade no panorama acadêmico, uma vez que têm crescido em número e complexidades de organização (BRENNAN; HACKLER; HOENE, 2005; BELL e JAYNE, 2009; VARNA; ADAMS; DOCHERTY, 2020). Este fenômeno tem sido catalis ado pelo êxodo rural e pelo interesse dos habitantes de grandes centros em migrarem para regiões menos pressionadas e caóticas, melhorando a qualidade de vida (BELL e JAYNE, 2009; HIGGINS e CAMPANERA, 2011). Vale ressaltar que, nesta pesquisa, considera-se cidade de pequeno porte aquela que tem população de até 50.000 habitantes, conforme explicitam Brennan; Hackler; Hoene (2005).

Por conta do tamanho diminuto, áreas urbanas de pequeno porte são unidades territoriais que demandam produtos de geoinformação em escala apropriada para que se façam ef etivas as políticas de gestão territorial (WOODCOCK e STRAHLER, 1987; ALVES et al., 2009; MARSHALL, 2011; CAMBOIM; BRAVO; SLUTER, 2015; SLUTER et al.., 2018; VARNA; ADAMS; DOCHERTY, 2020). Em contrapartida, é exatamente nessas regiões que se tem os maiores problemas de investimento no mapeamento de base (CAMBOIM; BRAVO; SLUTER, 2015; SLUTER et al., 2018). Especificamente no Brasil, a escassez de dados geográficos em zonas rurais e em pequenos aglomerados urbanos ocorre em f unção da falta de investimento público na criação de bases de dados espaciais (CAMBOIM; BRAVO; SLUTER, 2015; Sluter et al., 2018). É uma realidade observada e que preocupa desde a década de 1970 (BARRETO, 1970; LISBOA, 1971); é, ainda, um problema que precisa ser resolvido para que haja desenvolvimento ordenado. Nesse sentido, é importante buscar alternativas de baixo-custo que atendam à demanda de se observar sistemas urbanos pequenos, pois essas regiões sofrem com a falta de recursos financeiros para acessar a tecnologia que dê suporte ao ordenamento territorial (CAMBOIM; BRAVO; SLUTER, 2015; SLUTER et al., 2018; BOEING, 2019).

À guisa dessa necessidade, na década de 2000, a popularização do uso dos computadores pessoais (PCs) e da Internet (CASTELLS, 2009), impulsionou a facilitação de acesso aos produtos carto gráficos (HEIPKE, 2010; ROSA, 2011; GRIFFIN e FABRIKANT, 2012). Essa condição abriu oportunidade para o desenvolvimento de soluções tecnológicas que supram a carência por acessibilidade à geoinformação de regiões como os sistemas urbanos pequenos, o que amplia a possibilidade de se contornar o problema da lacuna tecnológica.

Nesse contexto, as imagens de satélites são produtos de geoinformação importantes ao reconhecimento de feições e fenômenos geográficos (LONGLEY, 2002; ROSA, 2011; JENSEN, 2014;). Com o avanço das tecnologias de informação relatado anteriormente, houve a facilitação do acesso e ao process amento de imagens obtidas em plataformas orbitais (LONGLEY, 2002; ROSA, 2011; GRIFFIN e FABRIKANT, 2012; FERSTER e COOPS, 2013; JENSEN, 2014; LLILESAND et al.., 2015). Paralelamente, o surgimento de softwares livres e aplicações centradas nos usuários, ainda na primeira década de 2000, também colaborou para o cenário atual de difusão de uso de produtos do sensoriamento remoto (HEIPKE, 2010; FERSTER e COOPS, 2013). Especificamente para o caso de estudos urbanos, é possível afirmar que o uso de imagens de satélite se disseminou por conta da interface que esses produtos oferecem à observação dos fenômenos espaciais, que podem ocorrer em escalas individualizadas e/ou mais amplas, com uma perspectiva integralizada, conectada (WOODCOCK e STRAHLER, 1987; LONGLEY, 2002; HEROLD; CLOUCLELIS; CLARKE, 2005; ALVES et al., 2009; YANG, 2011). Importante pontuar que, conforme explicam Herold; Clouclelis; Clarke (2005), as pesquisas que usam o sens oriamento remoto para observar áreas urbanas devem ir para além da simples aplicação de técnicas de classificação: precisam discutir os processos urbanos mediante a evolução espacial, temporal e dos relacionam entos dos fenômenos que integralizam a cidade. É uma discussão que encontra suporte em pesquisadores como Harvey (2000) e Corrêa (1989). Adiciona-se a esse ponto a necessidade de se criar modelos alternativos para observação de áreas urbanas de pequeno porte, utilizando tecnologia acessível aos gestores, para que se preencha a lacuna de acessibilidade tecnológica postulada anteriormente.

Considerando esse cenário, elaborou-se a presente pesquisa, cujo objetivo reside em analisar a evolução da área urbana de Monte Carmelo, MG. Selecionou-se Monte Carmelo como estudo de caso, pois essa é uma cidade de pequeno porte que sofre com a falta de dados espaciais, situação que impacta

\begin{tabular}{|c|c|}
\hline$C$ & Uberlândia-MG \\
\hline
\end{tabular}


negativamente a construção de políticas efetivas de gestão territorial. Entretanto, a maior motivação deste para a confecção deste estudo é balizada pela hipótese de que a evolução da paisagem urbana carmelitana tem fortes vínculos com a instalação do campus da Universidade Federal de Uberlândia (UFU) na cidade. O campus, nesse caso, serviria como vetor de atração à expansão urbana e, ao mesmo tempo, ag ente catalisador de processos urbanos em outras regiões da cidade. Essa hipótese encontra respaldo na pesquisa de Paula e Faria (2020).

Dessa maneira, para que se ating isse o objetivo maior e se testasse a hipótese levantada, desenvolveu-se um procedimento que automatiza a detecção da mancha urbana através do processo de segmentação de imagens multiespectrais do satélite RapidEye. Com este procedimento executado foi possível analis ar o fenômeno objeto de estudo segundo as particularidades das métricas espaciais (HEROLD; CLOUCLELIS; CLARKE, 2005), aqui materializadas no que se chamou de "adensamento urbano" e "expansão de fronteira". No primeiro caso, considera-se a expansão de fronteira urbana um ef eito de borda, i.e., um ef eito causado pela expansão do perímetro ocupado por habitações, vias e demais feições urb anas. Em complemento, o fenômeno do adensamento urbano é entendido neste artigo enquanto um ef eito ligado à densidade de ocupação do perímetro urbano já estabelecido.

\section{ÁREA DE ESTUDO}

Na Figura 1 representou-se a rede de vias urbanas do município de Monte Carmelo, cuja amplitude territorial auxilia na visualização da área e do fenômeno estudado.

Monte Carmelo é um município da mesorregião do Triângulo Mineiro e Alto Paranaíba, do Estado de Minas Gerais. Segundo o Instituto Brasileiro de Geografia e Estatística (IBGE) Monte Carmelo (g entílico "carmelitano") tem, aproximadamente 48.000 habitantes, com maioria residindo na área urbana (IBGE, 2019). Essa informação qualifica Monte Carmelo como um município de aglomeração urb ana pequena (BRENNAN; HACKLER; HOENE, 2005), o que atende a premissa do estudo de caso desse artigo.

Figura 1 - Localização da área de estudo. Destaque para as vias que atendem a cidade de Monte Camelo.

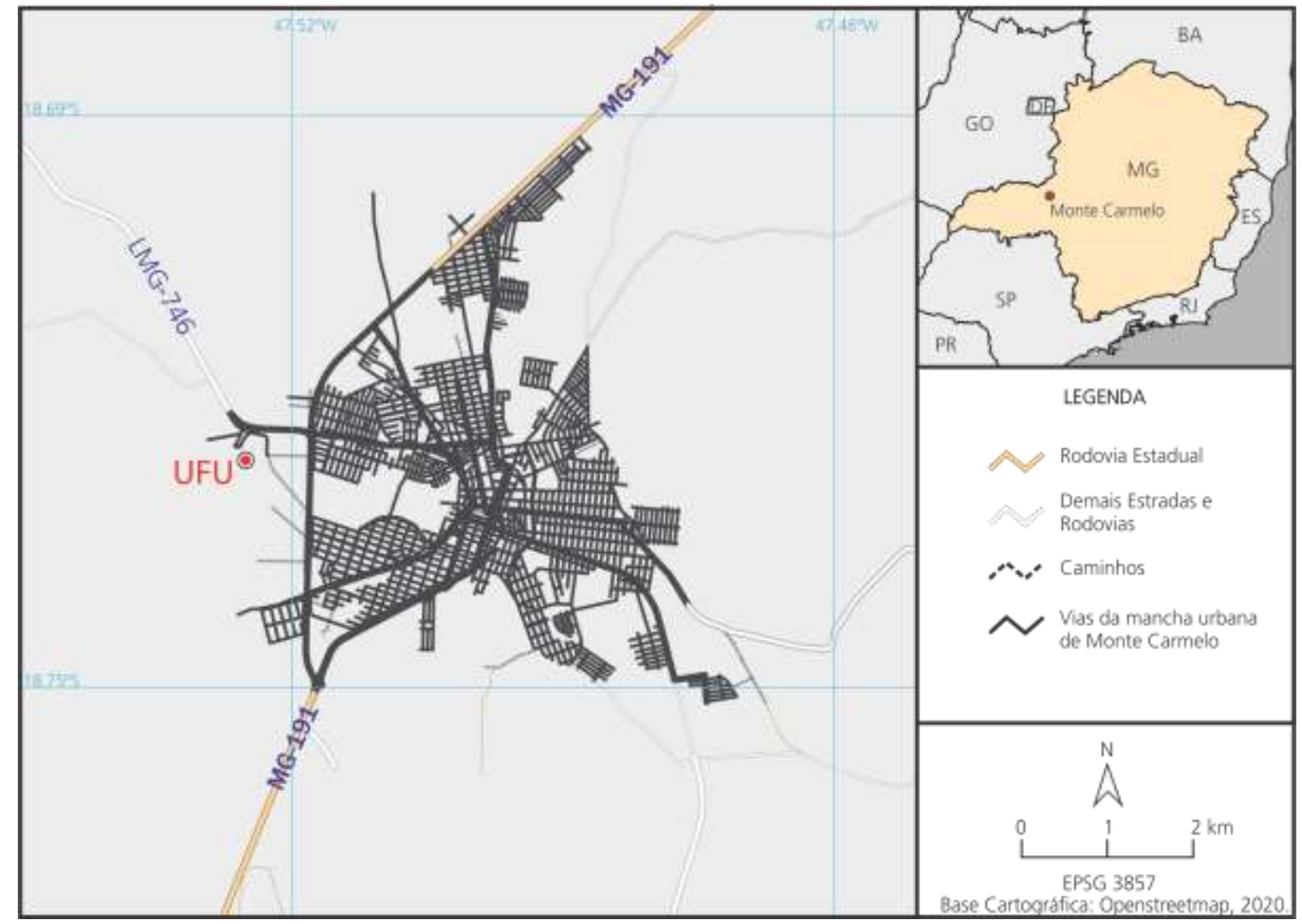

Fonte - Adaptado de Openstreetmap, 2020

$\begin{array}{llll}\text { Caminhos de Geografia } \quad \text { Uberlândia-MG } & \text { v. 22, n. } 82 \text { ago./2021 }\end{array}$

p. $28-40$

Página 30 
Monte Carmelo tem na agricultura e no comércio as principais atividades econômicas. Essa lo calidade é pólo regional do RIDES (Região Integrada de Desenvolvimento), servindo de referência nos serviços médicos e atividades comerciais aos 7 municípios do entorno, cuja abrangência po pulacional eng loba mais de 100.000 pessoas. É, portanto, na área urbana de Monte Carmelo que ocorrem muitos dos serviços essenciais para uma relevante parcela da população regional. Adicionalmente, Monte Carmelo é uma das cidades mineiras que recebeu um campus da Universidade Federal de Uberlândia, no ano de 2011. Como neste artigo trabalha-se com a hipótese de que a chegada da UFU favoreceu a mod ificação da dinâmica de ocupação do espaço urbano local, torna-se relevante compreender a dinâmica de evolução dessa região. Assim, esse estudo qualifica-se enquanto uma contribuição à gestão dos recursos e para o ordenamento territorial adequado às demandas que pressionam essa localidade.

\section{MÉTODO}

A sequência procedimental adotada neste estudo foi construída em três momentos principais, a saber, (1) a coleta e o pré-processamento das imagens, (2) os procedimentos para a detecção do fenômeno observado e (3) a análise dos resultados. Na primeira etapa, faz-se as correções necessárias à padronização das imagens e, na segunda etapa, aplica-se processos computacionais que destaquem a evolução do espaço urbano de Monte Carmelo. A terceira última etapa do método mo stra qual a ló gica adotada para se analisar os produtos gerados. Os itens que seguem mostram, em detalhe, os procedimentos em cada um dos momentos da pesquisa.

\section{COLETA E PRÉ-PROCESSAMENTO DAS IMAGENS}

Na primeira etapa, considerou-se que as imagens utilizadas neste estudo deveriam atender às demand as relacionadas à disseminação do uso de tecnologias da geoinformação para cidades pequenas, i.e., localidades com recursos escassos. As imagens deveriam permitir, ainda, a detecção dos movimentos da evolução urbana da cidade estudo de caso, i.e., com resoluções temporal, espectral e espacial adequadas à observação dos fenômenos de adensamento urbano e de expansão de fronteira. Assim, considerand ose as premissas elencadas, optou-se pela utilização de imagens do satélite RapidEye, gratuitas à finalidade de pesquisa quando solicitadas por órgãos e entidades federais ao Ministério do Meio Ambiente. Essa última condição preenche a premissa do desenvolvimento de uma solução de baixo-custo nesta pesquisa.

O sistema satelital RapidEye foi lançado em 2008 e é composto por uma constelação de cinco satélites de observação terrestre. Cada um destes satélites está posicionado em órbita heliossíncrona, com igual espaçamento entre plataformas. O sistema de satélites RapidEye conseguem contornar o planeta Terra 15 vezes por dia, imageando, aproximadamente, 4,5 milhões de $\mathrm{km}^{2} / \mathrm{dia}$. Nessas condições, o tempo de revisita no modo nadir (visada ortogonal) é de 5,5 dias e, no modo off-nadir (visada oblíqua) a revisita é diária. O sensor embarcado nos satélites desta constelação é do tipo pushbroom, i.e., scanner de linha, capaz de capturar imagens em cinco bandas espectrais. As imagens capturadas representam a superfície terrestre com resolução espacial original de $6,5 \mathrm{~m}$ e, após a ortorretificação, aplica-se um processo de reamostragem que, supostamente, entrega um produto com resolução espacial de $5 \mathrm{~m}$. Tais sensores captam dados com resolução radiométrica de 12 bits (FELIX; KAZMIERCZAK; ESPINDOLA, 2009). Essas condições demonstradas revelam que as imagens do satélite RapidEye são sensíveis à detecção dos fenômenos que são observados nesta pesquisa, uma vez que atendem ao demandado em termos de resolução espacial, temporal e espectral.

Especificamente, as imagens multiespectrais RapidEye desta pesquisa foram obtidas a partir da plataforma "GeoCatálogo" do Ministério do Meio Ambiente. Adquiriu-se somente imagens capturadas na posição nadir, para que não houvesse oclusão de alvos ou perda de detalhamento nas bordas, condições que poderiam afetar a sensibilidade dos processos de detecção de crescimento. Todas as imagens foram obtidas em épocas do ano semelhantes, para que se mantive a quantidade de radiação solar disponível equivalente.

Assim, a avaliação da evolução urbana de Monte Carmelo ocorreu mediante a delimitação de um es paço temporal representativo à avaliação. Dessa maneira, selecionou-se os anos de 2013 a 2018. Do is anos antes desse período houve a instalação da Universidade Federal de Uberlândia na cidade de Monte Carmelo, lacuna que permitiu medir as modificações no espaço urbano segundo o início ef etivo das atividades acadêmicas da UFU.

\begin{tabular}{|c|c|}
\hline $\mathrm{C}$ & Uberlândia-MG \\
\hline
\end{tabular}


Em posse das imagens, seguiu-se para o pré-processamento. Na etapa de pré-processamento fez-se as correções radiométricas e geométricas das imagens. Essas correções visam melhorar as cond iç ões de observação dos alvos imageados e dar equivalência às comparações, assim como as feitas na análise multitemporal desta pesquisa (JENSEN, 2014; GONZALEZ e WOODS, 2007; LILLESAND; KIEFER; CHIPMAN, 2015).

Sequencialmente, a primeira etapa do pré-processamento é o registro das imagens. Nesse procedimento se faz a correção geométrica dos produtos. A operação de registro estabelece uma transformação geométrica entre espaços, pois sabe-se que as condições de aquisição são diferentes para cada época e, embora os produtos da constelação RapidEye seja a única fonte de análise deste es tudo, é importante reconhecer que existem perturbações no momento de tomada das imagens, as quais impactam na geometria da cena imageada (LILLESAND; KIEFER; CHIPMAN, 2015). Nesse caso, como este estudo se vale de uma avaliação multitemporal, é importante reconhecer uma das imagens como referencial à transformação. Dessa forma, optou-se pela imagem de 2017, determinando-se alvos detectáveis em todas as imagens restantes. No processo de registro preocupou-se, ainda, com a geometria dos pontos de controle do registro, para que os erros das transformações fossem distribuídos sem tendências nas imagens transformadas (JENSEN, 2014; LILLESAND; KIEFER; CHIPMAN, 2015).

Depois do processo de registro, a transformação que segue está relacionada aos valores digitais das imagens (GONZALEZ e WOODS, 2007). São duas as principais etapas, a saber, a correção atmosférica e a normalização radiométrica. A correção atmosférica é uma transformação algébrica que altera os números digitais da imagem original para que haja remoção de condições que perturbam a resposta espectral dos alvos por influência da camada atmosférica entre o sensor e os alvos. São efeitos conhecidos, por exemplo, a absorção e espalhamento atmosférico (JENSEN, 2014; LILLESAND; KIEFER; CHIPMAN, 2015). Com a correção atmosférica feita os números digitais são transformados de um estado "bruto" para valores com significado físico, como a reflectância de superfície (JENSEN, 2014; LILLESAND; KIEFER; CHIPMAN, 2015).

Para realização da correção atmosférica - e das demais etapas deste estudo - utilizou-se o software Envi, com licença concedida à Universidade Federal de Uberlândia. Os procedimentos para a correção foram a implementados na rotina FLAASH, a qual é indicada para imagens multiespectrais de média e alta resolução espacial (COOLEY et al., 2002; YUAN e NIU, 2008; PERKINS, 2012). Para utilização deste método foi necessário conhecer os comprimentos de onda de cada uma das imagens de entrada e indicálos no cabeçalho dos arquivos. Após esse procedimento converteu-se a imagem para o formato Bil, o qual é definido como formato padrão para ativar o módulo de correção FLAASH. Além da padronização de formatos de leitura, alguns parâmetros foram informados para ajustar o modelo "emp írico" de co rreção atmosférica implementado no módulo FLAASH. Para tanto, definiu-se um fator de escala 1000 para tod as as imagens corrigidas, especificado o tipo de sensor, a data e horário de aquisição da imagem, altitude do terreno, coordenada central da cena, tamanho do pixel e altitude do sensor. Ressalta-se que estes procedimentos encontram respaldo nos trabalhos de Cooley et al. (2002), Yuan e Niu (2008) e Perkins (2012).

Após a correção atmosférica, fez-se a normalização radiométrica. A normalização radiométrica visa garantir a homogeneidade radiométrica do conjunto de imagens multitemporais (JENSEN, 2014; LILLESAND; KIEFER; CHIPMAN, 2015; RAMIREZ; ANDERSON, FORMAGGIO, 2015). Esse procedimento foi realizado com uma metodologia multivariada de normalização de intervalos, tendo uma imagem como referência para a normalização das demais. Nesta ocasião a imagem de 2017 foi utilizada, novamente, como referência. A normalização foi executada banda a banda em todas as imagens, processo no qual definiu-se uma função individual de normalização para os distintos canais.

\section{PROCEDIMENTOS PARA DETECÇÃO DA EVOLUÇÃO URBANA DE MONTE CARMELO}

O primeiro processo aplicado à deteç̧ão das feições urbanas foi o de segmentação das i magens. Após avaliar visualmente a resposta espectral da área urbana nas cinco bandas (Vermelho, Verde, Azul, Infravermelho próximo), percebeu-se que alvos relacionados à evolução urbana destacavam-se na banda do vermelho. A partir deste momento, utilizou-se apenas as imagens referentes a essa faixa do espectro

\begin{tabular}{|c|c|}
\hline Caminhos de Geografia & Uberlândia-MG \\
\hline
\end{tabular}


eletromagnético. Deve-se ressaltar que esta escolha é oportuna, uma vez que, alta resposta do concreto e do solo exposto e a baixa resposta da vegetação neste comprimento de onda (JENSEN, 2014). Assi m a banda do vermelho foi utilizada para aplicação da operação de segmentação, descrita pela equação 1.

$$
E s=\sum \frac{x_{\bar{i}}}{n}+\sqrt{\sum \frac{\left(x_{\bar{i}}-\bar{x}\right)^{2}}{n-1}}
$$

Onde:

$\mathrm{xi}$ = i-ésimo pixel da banda vermelha;

$\mathrm{n}=$ número de pixels;

$\bar{X}=$ média dos pixels na banda vermelha

Es = Equação de segmentação de áreas urbanas;

Através da análise dos histogramas das imagens (GONZALEZ e WOODS, 2007) para os diferentes anos, observou-se que a resposta espectral das feições da área urbana concentrava-se em uma faixa específica de brilho, a saber, um sigma (desvio padrão) "positivo" da média dos valores no histograma. Assim, após essa constatação formalizou-se a separação de classes via a operação histogrâmica, discriminand o-se a área urbana das demais feições presentes na cena. Após essa operação, binarizou-se as imagens resultantes para que se obtivesse um produto que destacasse a mancha urbana dos demais alvos (GONZALEZ e WOODS, 2007; LILLESAND; KIEFER; CHIPMAN, 2015).

Para que fossem quantificadas as diferenças entre as imagens, realizou-se a detecção de mudanças por meio de operações algébricas. Assim pôde-se observar a dinâmica evolutiva do fenômeno de expansão urbana de Monte Carmelo, contabilizando-se a área ocupada pelas feições urbanas ano a ano. Especificamente, as referidas operações algébricas foram materializadas na subtração de imagens, procedimento feito aos pares de imagens, e.g., da imagem do ano de 2018 subtraiu-se a imagem de 2017. Como resultado se tem as diferenças nos valores de áreas atribuídos à "movimentação" das feições urbanas.

\section{ANÁLISE DOS RESULTADOS}

$\mathrm{Na}$ análise dos resultados observou-se os produtos resultantes das operações de segmentação descritas no item anterior. Além disso, fez-se a contabilização dos valores em área atribuídos às feições urb anas e fez-se uma avaliação visual comparativa. Essa avaliação visual possibilitou que se discriminasse qualitativamente os reais motivos da evolução urbana, separando-se a condição de "adensamento urbano" da condição de "expansão de fronteira".

\section{RESULTADOS E DISCUSSÕES}

A Figura 2 mostra o resultado do processo de segmentação que deu origem à primeira fase de análises, a saber, a análise visual. Na Figura 2 visualiza-se os trechos urbanos destacados em branco e as demais feições em preto. A Figura 3 complementa a compreensão da dinâmica evolutiva ao demonstrar o total de habitantes de Monte Carmelo, desde o ano da chegada a UFU à cidade. Importante destacar que, nes te estudo, se associa o incremento na população verificado no período de 2012 e 2013 (Figura 3) à instalação e do início das atividades do campus Monte Carmelo, da Universidade Federal de Uberlândia.

Nesse contexto, na Figura 2 é possível distinguir os dois processos estudados neste artigo: a expansão de fronteira e o adensamento urbano. No primeiro caso, é possível verificar que Monte Carmelo não expandiu suas fronteiras urbanas de maneira agressiva, considerando-se o intervalo temporal analisado nesta pesquisa. Tal afirmação fundamenta-se na ideia de que se manteve as principais formas da cidade, conf orme discute Corrêa (1989). Essa constatação demonstra que, apesar do crescimento populacio nal projetado pelas estimativas do IBGE no intervalo dos anos de 2012 e 2013 (Figura 2), a taxa de ocupação de novas áreas não foi acelerada, em virtude do espaço ocioso que já exis tia na cidade. Esse espaço ocioso estava reservado à criação de condomínios e loteamentos. Tais regiões podem ser observadas na Figura 4 (em "A", "B", "C", "D" e "E"), mais adiante.

\begin{tabular}{|c|c|}
\hline $\mathrm{C}$ & Uberlândia-MG \\
\hline
\end{tabular}


Figura 2 - Imagens resultantes do processo de segmentação.

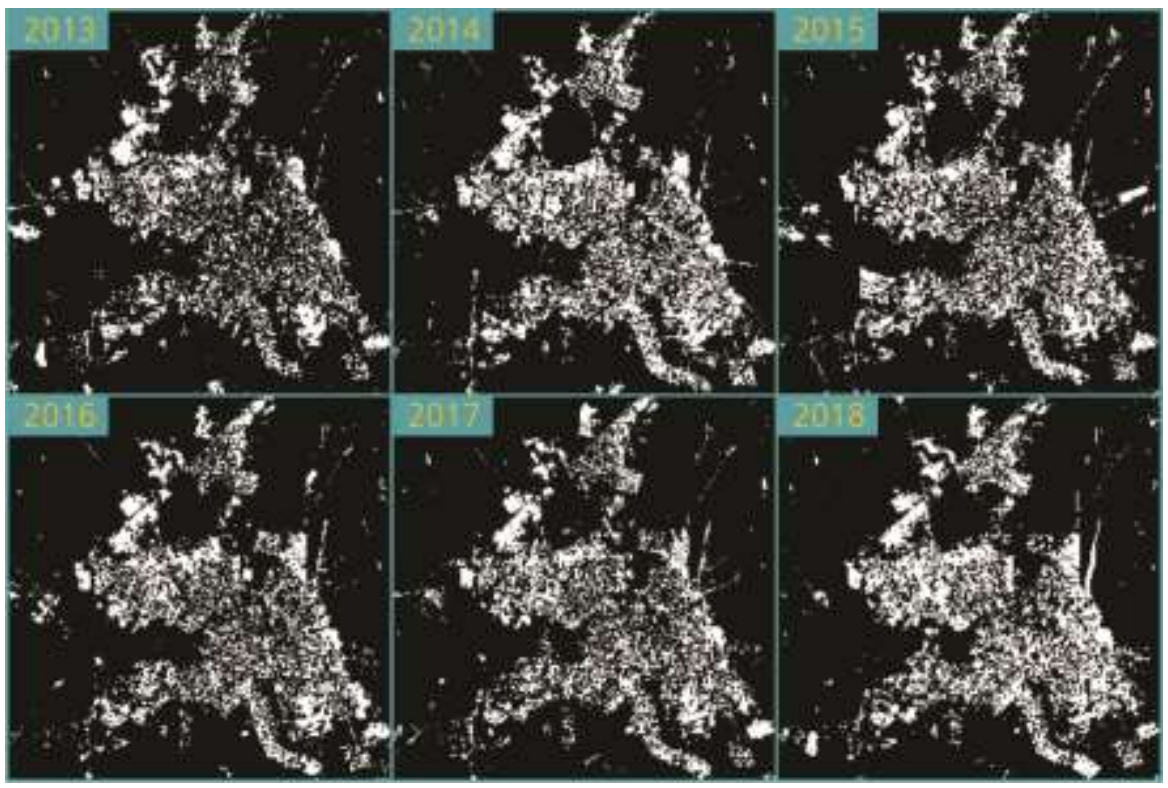

Figura 3 - Crescimento populacional de Monte Carmelo, estimado pelo IBGE.

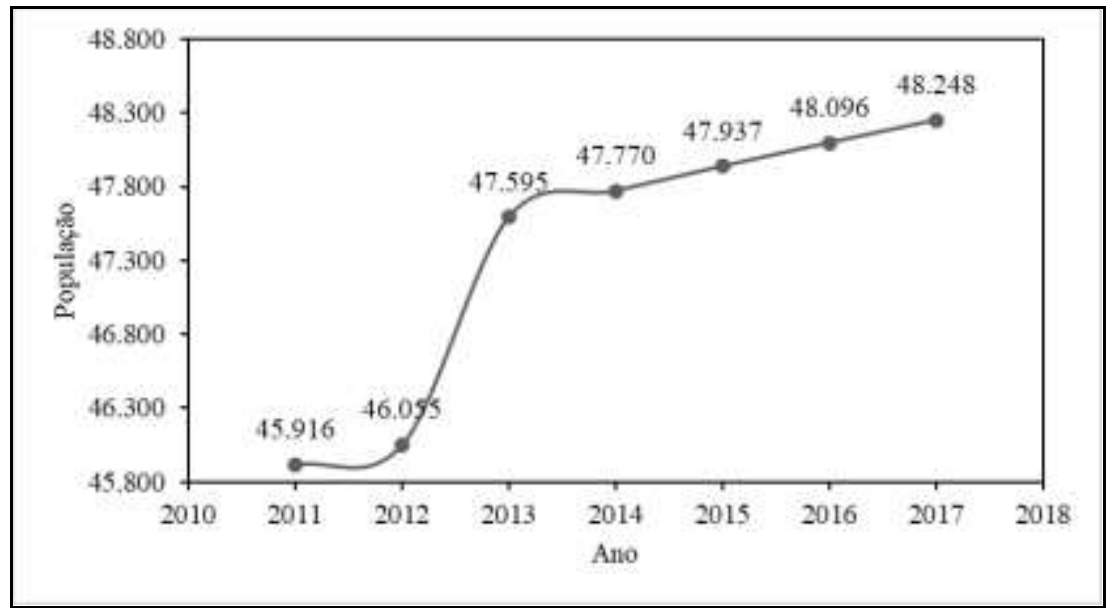

Fonte - IBGE (2019).

Figura 4 - Áreas em destaque para a explicação da expansão de fronteira urbana e do adensamento urbano de Monte Carmelo.

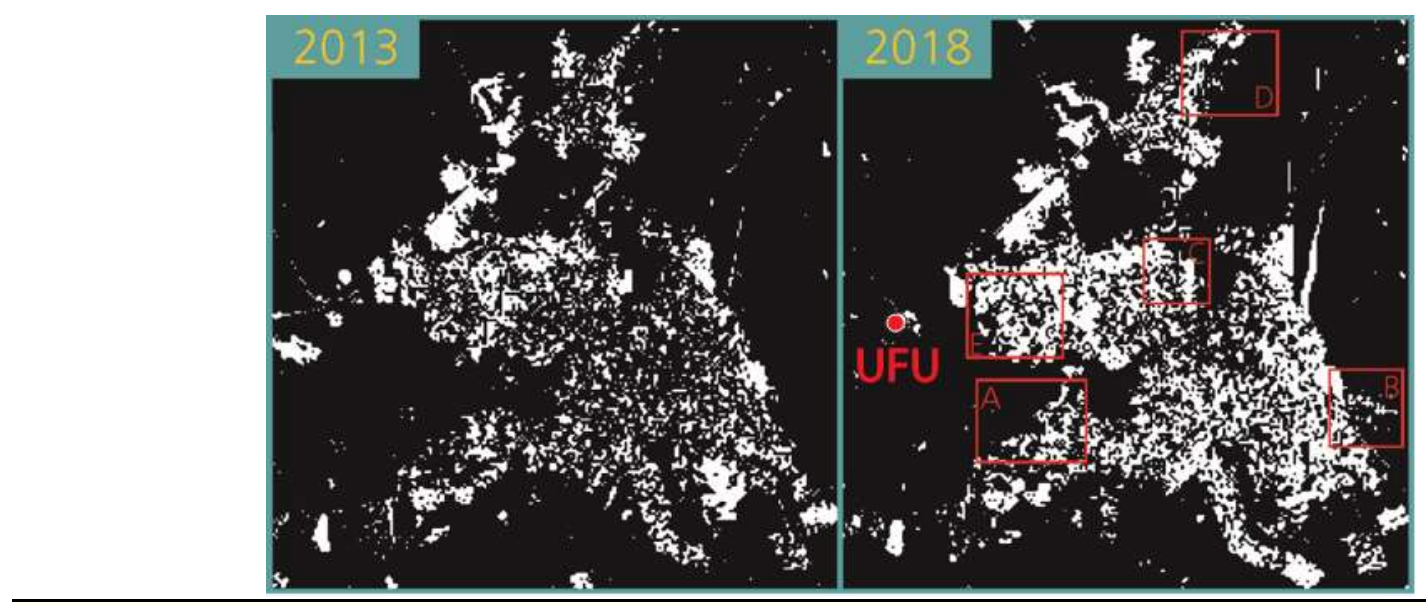

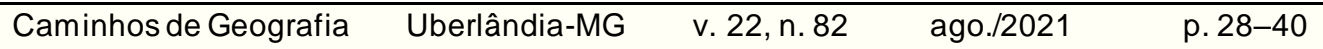


Uma análise das consequências da implantação do campus da Universidade Federal de Uberlândia na evolução urbana de Monte Carmelo por meio de imagens multiespectrais de baixo custo
Alexandre Fleury de Siqueira e Gomes George Deroco Martins João Vitor Meza Bravo

Com ef eito, Corrêa (1989) discute que os segmentos de ocupação das cidades na América latina seguem o padrão no qual as zonas periféricas e desprovidas da aparelhagem urbana ideal, são preenchid as por populações mais pobres. Quando se observa a distribuição de renda relacionada à população conectada às atividades da UFU, em especial, os servidores, é de esperar que tais indivíduos não p rocurem áreas periféricas, demasiadamente afastadas da aparelhagem da cidade ou do próprio local de trabal ho: o que confirmaria a lógica apontada por Corrêa (1989). Nesse sentido, ao se analisar a Figura 4, afirma-se que somente estão associadas à expansão de fronteiras as áreas "B" e "D", com loteamentos de o cup ação recente. São setores cuja distância do aparelho citadino carmelitano e do campus da Universidade Federal de Uberlândia, permite relacionar o processo de ocupação a outros segmentos de habitantes que não aqueles ligados à UFU. São áreas que fazem limite direto com propriedades rurais, diferente da situação das demais áreas destacadas. Dessa forma, pode-se dizer que há o indício de predominância de um padrão de ocupação estruturado pela chegada do campus da UFU a Monte Carmelo. A Figura 5 permite a visualização das áreas destacadas na Figura 4: são fotos que registram as condições da paisagem local, nas quais há presença de loteamentos recentes, fato que corrobora os argumentos expostos.

Figura 5 - Fotos de loteamentos em áreas de expansão de fronteira, ano de 2018.
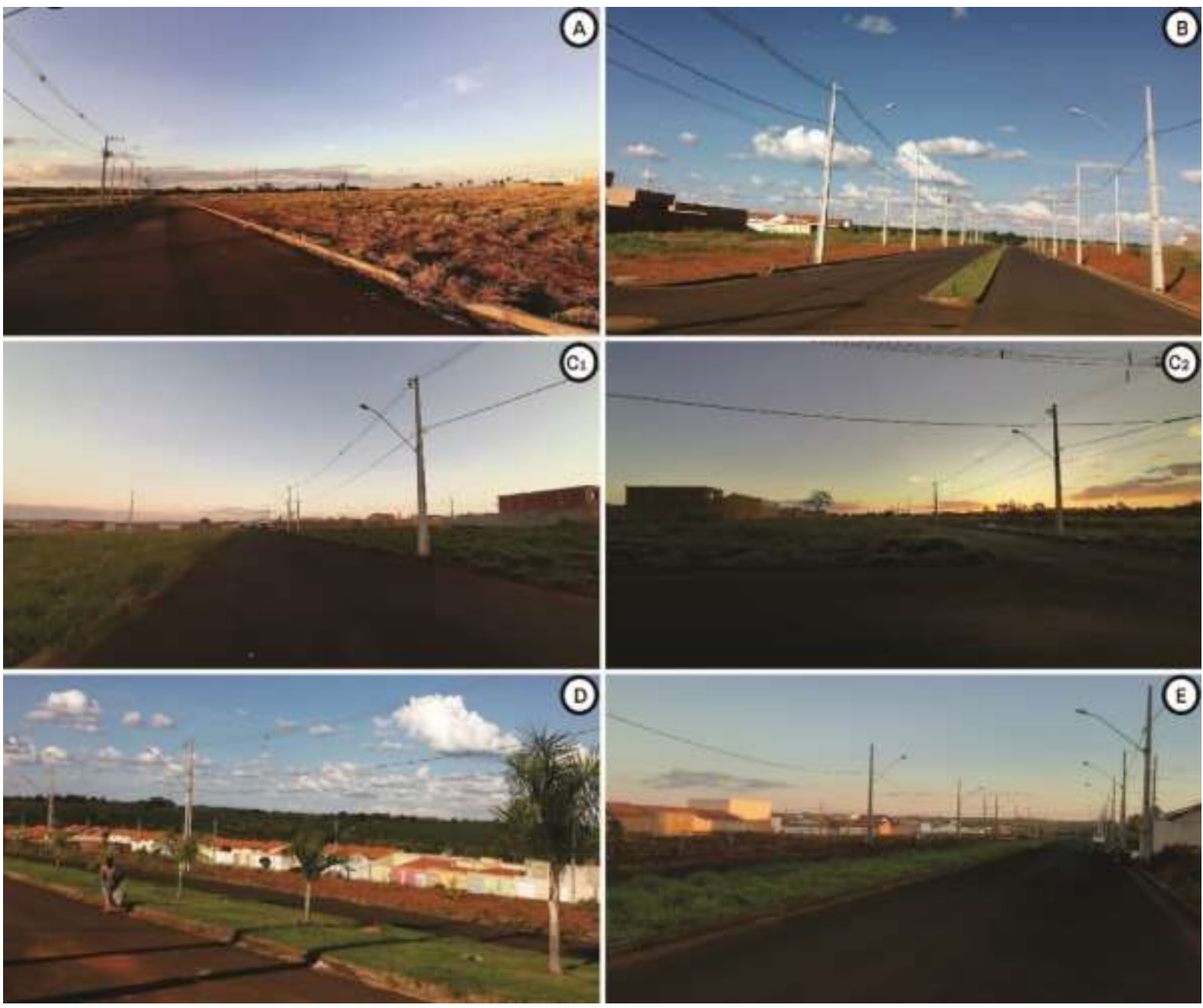

Embora a expansão da fronteira urbana de Monte Carmelo tenha ocorrido de modo contido, é de se esperar que o aumento populacional tenha gerado impactos nas características urb anas lo cais. Desse modo, a condição de pouca expansão de fronteiras observada abre marg em à interpretação de outro fenômeno, o do adensamento urbano. Assim, considera-se conexões causais ao adensamento urb ano carmelitano a construção de vias, como a Avenida Brasil Oeste, a conexão entre o bairro Jardim dos Ipês

$\begin{array}{llllll}\text { Caminhos de Geografia } & \text { Uberlândia-MG } & \text { v. 22, n. 82 } & \text { ago./2021 } & \text { p. 28-40 } & \text { Página } 35\end{array}$


e bairro do Carmo, a criação de loteamentos e, em especial, as atividades da Universidade Fed eral de Uberlândia (Figura 6).

Ao revisitar a Figura 4, é possível notar o claro adensamento urbano nas áreas destacadas em " $\mathrm{A}$ ", " $\mathrm{C}$ " e "E"; item confirmado nas imagens das Figuras 5 e 6 . Nesses setores de loteamentos também recentes há um marcador importante que impede que haja a expansão da fronteira urbana: a rodovia MG-191. Dessa forma, só resta a essas áreas o adensamento da massa de imóveis e das vias urbanas. Destaque especial pode ser dado à área $E$ (Figuras 4 e 5, trecho também destacado na Figura 6), pois há maio r proximidade com o campus Monte Carmelo da Universidade Federal de Uberlândia. Nesse setor residem, principalmente, estudantes e professores da UFU. A acolhida aos indivíduos vinculados à UFU é processo semelhante ao que ocorre nas áreas A e C (Figura 4), apesar da intensidade menor nesses locais. Essa é uma importante constatação, uma vez que ratifica o caráter transformador das universidades, quand o, na interiorização dos campi, retrabalha-se a estrutura citadina próxima aos locais em que se instalam as instituições (PAULA e FARIA, 2020).

Figura 6 - Contexto dos investimentos estruturais e imobiliários que suportam o raciocínio do adensamento urbano.

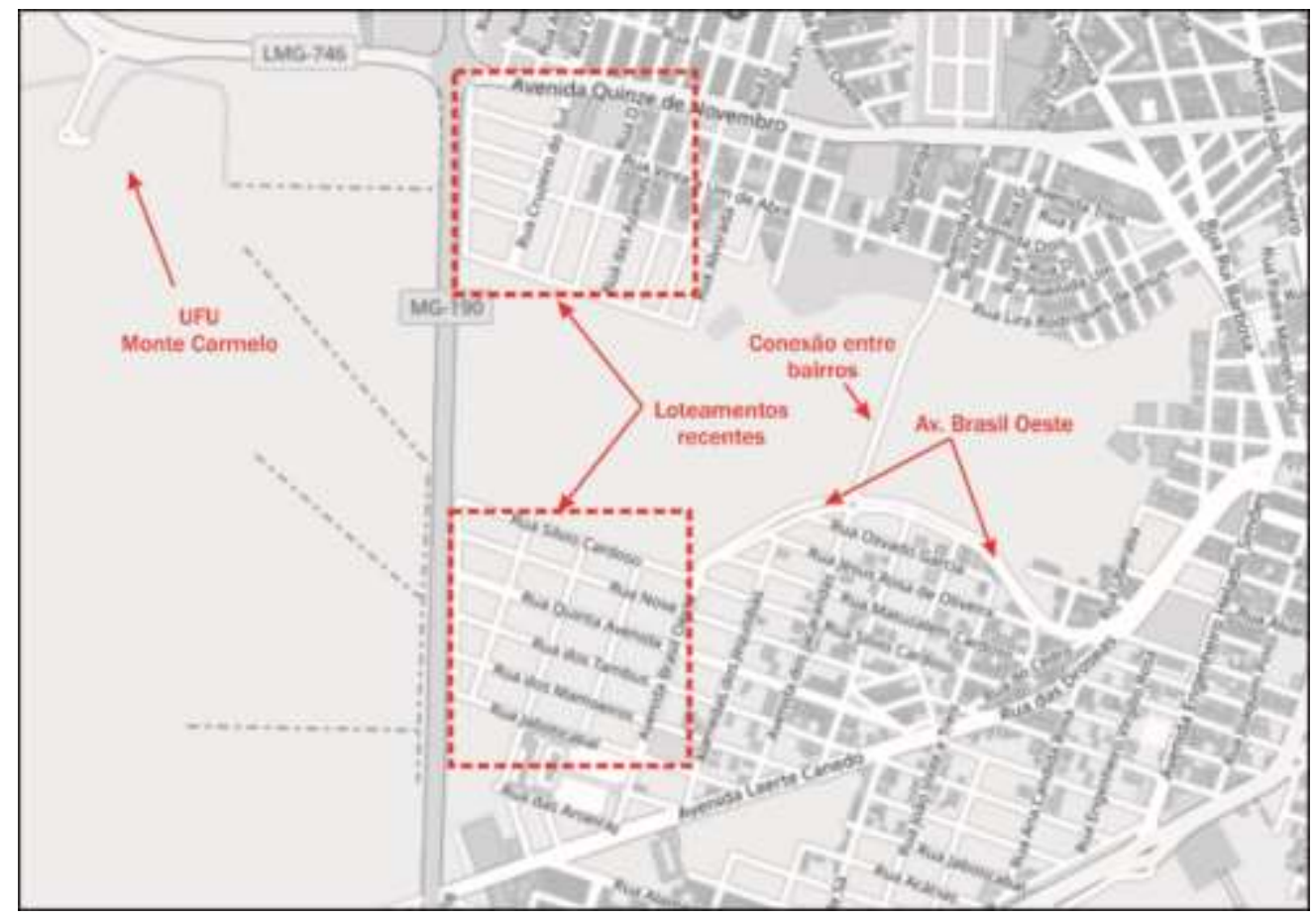

Adaptado de OpenStreetMap, 2020.

Em termos numéricos, é possível perceber que as hipóteses levantadas na análise visual se confirmam. Nesse sentido, a Tabela 1 mostra a evolução dos valores de área ocupada pelas feições urbanas de Monte Carmelo. Após o processo de segmentação, contabilizou-se a área em metros quadrados e a quantidade de pixels classificados como feições urbanas. A comparação numérica da ev olução fo i feita observando-se a diferença anual na área ocupada por feições urbanas.

Tabela 1 - Medidas da evolução de ocupação espacial dasfeições urbanas de Monte Carmelo.

\begin{tabular}{ccccc}
\hline Intervalo temporal & Diferença (anos) & Área (pixels) & Área $\left(\mathbf{m}^{2}\right)$ & Crescimento(\%) \\
\hline $\mathbf{1}$ & $2014-2013$ & 207849 & 1039245 & 10,5104 \\
$\mathbf{2}$ & $2015-2014$ & 92364 & 461820 & 4,6705 \\
$\mathbf{3}$ & $2016-2015$ & 197168 & 985840 & 9,9702 \\
$\mathbf{4}$ & $2017-2016$ & 71707 & 358535 & 3,6261 \\
$\mathbf{5}$ & $2018-2017$ & 212603 & 1063015 & 10,7507
\end{tabular}


Uma análise das consequências da implantação do campus da Universidade Federal de Uberlândia na evolução urbana de Monte Carmelo por meio de imagens multiespectrais de baixo custo
Alexandre Fleury de Siqueira e Gomes George Deroco Martins João Vitor Meza Bravo

Na Tabela 1 é possível observar que há um crescimento gradual da área ocupada por feições urbanas em Monte Carmelo, após o início do ano de 2013. Novamente, justifica-se tal efeito pela instalação da Universidade Federal de Uberlândia, pela criação dos loteamentos e pelas obras de infraestrutura realizadas pela pref eitura municipal. Esse resultado encontra respaldo nas af irmações feitas por Paula e Faria (2020), que notaram processos semelhantes na cidade de Viçosa. Com efeito, para se confirmar que a criação do campus Monte Carmelo e suas atividades está conectada à evolução da área urbana carmelitana, bastaria observar os picos nas séries 1 , 3 e 5, da Tabela 1. Nesses intervalos temporais temse o início das atividades da UFU no campus Sesi, o início das atividades de novos cursos de grad uação e o início das atividades no campus mais afastado, no bairro Araras, respectivamente. A Figura 7 mostra em destaque a modificação trazida pelo campus Monte Carmelo, no bairro araras.

Figura 7 - A chegada do campus Monte Carmelo da Universidade Federal de Uberlândia (destaque em vermelho)e os impactos na área dos bairros próximos.

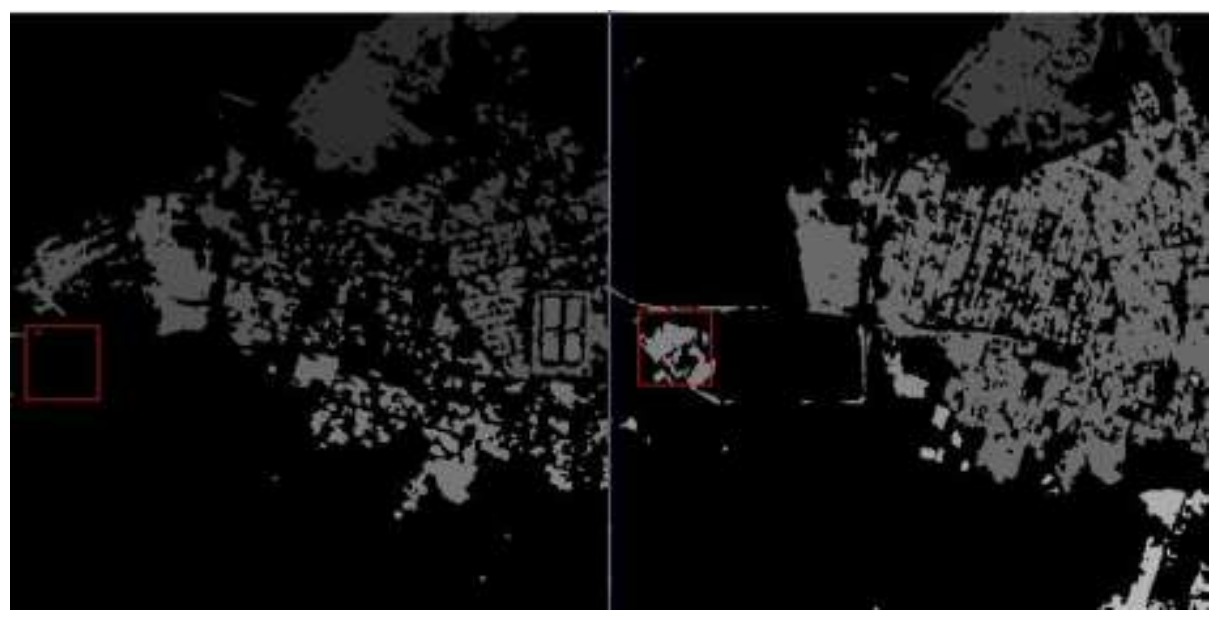

De especial importância para este trabalho, a Figura 8 mostra, em detalhe, as imediações do campus da UFU. Nessa figura é possível observar que o campus Monte Carmelo está localizado em uma área predominantemente agrícola. É, portanto, vetor que atrai o expansão urbana para o setor oeste da área urbana de Monte Carmelo.

Figura 8 - A UFU campus Monte Camelo em seu contexto geográfico.

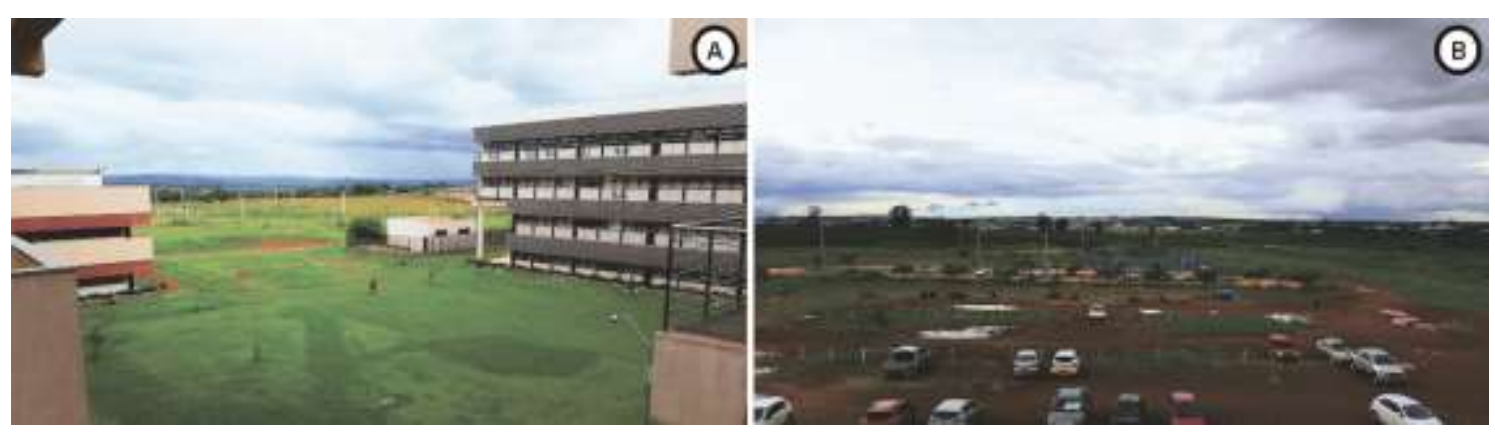

Ainda na Figura 8, em "A", tem-se destaque para a estrutura predial do campus. Paralelamente, em B temse destaque para o ponto de vista do observador do campus para a cidade de Monte Carmelo. Em "B" ainda é possível ver a reserva de espaço entre o campus e a cidade, ainda ocupado por um cafeeiro. Este setor poderá, no futuro, capitanear a maior parte dos avanços da área urbana carmelitana, caso se mantenha o padrão de movimentação observado neste artigo. Essa movimentação de expansão de fronteiras, por si, altera a forma da cidade (Corrêa, 1989), o que impacta na g es tão e no ord enamento territorial local.

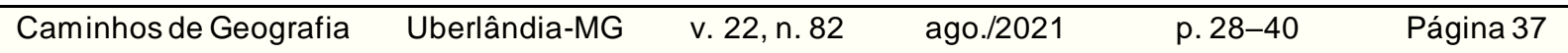




\section{CONCLUSÕES}

Neste artigo foi possível analisar a evolução da expansão urbana de Monte Carmelo com produtos de geoinf ormação de baixo-custo, o que impacta positivamente a acessibilidade tecnológica demandada por gestores de cidades pequenas. A avaliação feita nesse artigo ponderou que a expansão urbana desta localidade ocorreu em função de dois movimentos principais: a expansão de fronteiras, em menor proporção, e o adensamento urbano, em maior proporção.

Concluiu-se que o adensamento urbano carmelitano pode ser explicado pela chegada da Universid ade Federal de Uberlândia à cidade, especialmente com o início das atividades de ensino nos diferentes momentos. Isso pode ser afirmado pois os maiores crescimentos estão vinculados aos períodos de inicialização das atividades dos cursos e à construção do campus no bairro Araras. Es pecificamente, 0 impacto da instalação do campus universitário da UFU em Monte Carmelo ocorreu em função da contratação de docentes e da chegada de alunos para os cursos de graduação que iniciaram suas atividades em 2011. São mais de 1000 indivíduos vinculados à vida universitária da UFU Monte Carmelo, número que representa mais de $2 \%$ da população carmelitana. Postos esses argumentos, comprovou-se a veracidade da hipótese levantada incialmente.

Além da instalação da UFU em Monte Carmelo, discutiu-se sobre o impacto dos investimentos da prefeitura municipal - no tocante aos melhoramentos da infraestrutura da cidade (malha viária) - e dos empreendimentos imobiliários, em especial, loteamentos, na evolução do es paço urb ano carmelitano. Todavia, é importante evidenciar que estas obras foram executadas em função do aquecimento da economia local, em grande parte estimulado pela chegada a UFU à Monte Carmelo.

De todo modo, pode-se concluir que o crescimento populacional motivado, principalmente, pelas atividades da UFU foi variável que incitou o desenvolvimento urbano de Monte Carmelo, especialmente na área próxima ao campus. Além da modificação na estrutura urbana carmelitana, a instalação do campus da Universidade Federal de Uberlândia impactou positivamente a economia local com inserção de indivíduos economicamente ativos e com poder aquisitivo para movimentar o setor imobiliário. Essa dinâmica é semelhante àquela relatada por Paula e Faria (2020). Além dos impactos econômicos, se vê, também, que as ações de extensão promovidas pela UFU Monte Carmelo têm importante papel ao aproximar os membros da sociedade local da vida universitária e do conhecimento promovido à luz da universidade pública e gratuita.

\section{REFERÊNCIAS}

ALVES, C. D.; PEREIRA, M. N.; FLORENZANO, T. G.; MARCELHAS E SOUZA, Í. Análise orientada a objeto no mapeamento de áreas urbanas com imagens landsat. Boletim de Ciências Geodésicas, vol. 15 n.1, p. 120-141, 2009.

BARRETO, A. Mapeamento do Brasil a curto prazo. Revista Brasileira de Cartografia, vol.1, n. 1, 38-43, 1970.

BATTY, M.; MARSHALL, S. The Origins of Complexity Theory in Cities and Planning. In Portugali, J.; H. Meyer; Stolk, E.; Tan, E. (eds.). Complexity Theories of Cities Have Come of Age. Berlin: Springer, p. 21-45, 2011. https://doi.org/10.1007/978-3-642-24544-2 3

BELL, D.; JAYNE, M. Small Cities? Towards a Research Agenda. International Journal of Urban and Regional Research, vol. 33, n. 3, p. 683-699, 2009. https://doi.org/10.1111/j.1468-2427.2009.00886.x

BOEING, G. Spatial information and the legibility of urban form: Big data in urban morphology.

International Journal of Information Management, in press, 2019. https://doi.org/10.31235/osf.io/vhrdc

BRENNAN, C., HACKLER, D., HOENE, C. Demographic Change in Small Cities, 1990 to 2000. Urban Affairs Review, vol. 40, n. 3, p. 342-361, 2005. https://doi.org/10.1177/1078087404269161

BUHAUG, H.; URDAL, H. An urbanization bomb? Population growth and social disorder in cities. Global Environmental Change, vol. 23, n. 1, p. 1-10, 2013. https://doi.org/10.1016/j.gloenvcha.2012.10.016

CAMBOIM, S. P., BRAVO, J. V. M., SLUTER, C. R. An investigation into the completeness of, and the updates to, OpenStreetMap data in a heterogeneous area in Brazil. ISPRS International Journal of GeoInformation, vol. 4, n. 3, p. 1366-1388, 2015. https://doi.org/10.3390/ijgi4031366

\begin{tabular}{|c|c|}
\hline$C$ & Uberlândia-MG \\
\hline
\end{tabular}


CASTELLS, M. The Rise of the Network Society. Wiley-Blackwell, UK, 2009.

https://doi.org/10.1002/9781444319514

COOLEY, T.; ANDERSON, G. P.; FELDE, G. W.; HOKE, M. L.; RATKOWSKI, A. J.; CHETWYND, J. H.; GARDNER, J. A.; ADLER-GOLDEN, S. M.; MATTHEW, M. W.; BERK, A.;BERNSTEIN, L. S.; ACHARYA, P. K.; MILLER, D.; LEWIS, P. FLAASH, a MODTRAN4-based atmospheric correction algorithm, its applications and validation. In Proceedings of the International Geoscience and Remote Sensing Symposium (IGARSS), p. 1414-1418, 2002.

CORRÊA, R. L. O Espaço Urbano. Ática, São Paulo, 1989.

FELIX, I. M; KAZMIERCZAK, M. L.; ESPINDOLA G. M. RapidEye: a nova geração de satélites de observação da Terra. In: Anais do XIV Simpósio Brasileiro de Sensoriamento Remoto, p. 7619-7622, Natal, INPE, 2009.

FERSTER, C. J.; COOPS, N. C. A review of earth observation using mobile personal communication devices. Computers e Geosciences, vol. 51, p. 339-349, 2013.

https://doi.org/10.1016/j.cageo.2012.09.009

GONZALEZ, R. C.; WOODS, R. E. Digital Image Processing (3rd Edition). In Prentice-Hall, Inc. Upper Saddle River, NJ, USA, 2007.

GRIFFIN, A. L; FABRIKANT, S. I. More Maps, More Users, More Devices Means More Cartographic Challenges. The Cartographic Journal, vol. 49, n. 4, p. 298-301, 2012.

https://doi.org/10.1179/0008704112Z.00000000049

HARVEY D. Megacities Lecture 4: Possible Urban Worlds. Twynstra Gudde Management Consultants, Amersfoort, The Netherlands, 2000.

HEIPKE, C. Crowdsourcing geospatial data. ISPRS Journal of Photogrammetry and Remote Sensing, vol. 65, n. 6, p. 550-557, 2010. https://doi.org/10.1016/j.isprsjprs.2010.06.005

HEROLD, M.; COUCLELIS, H.; CLARKE, K. C. The role of spatial metrics in the analysis and modeling of urban land use change. Computers, Environment and Urban Systems, vol. 29, n. 4, p. 369-399, 2005. https://doi.org/10.1016/j.compenvurbsys.2003.12.001

HIGGINS, P.; CAMPANERA, J. M. (Sustainable) quality of life in English city locations. Cities, vol. 28, n. 4, p. 290-299, 2011. https://doi.org/10.1016/j.cities.2011.02.005

Instituto Brasileiro de Geografia e Estatística - IBGE. Estimativa da população. Rio de Janeiro: IBGE, 2014. Disponível em http://www.ibge.gov.br Acesso em: 3 de dezembro de 2019

JENSEN, J. R. Remote sensing of the environment: an earth resource perspective (second edition). In Pearson Education Limited,Harlow, England, 2014.

KANAI, J. M.; GRANT, R.; JIANU, R. Cities on and off the map: A bibliometric assessment of urban globalization research. Urban Studies, vol. 55, n. 12, p. 2569-2585, 2017.

https://doi.org/10.1177/0042098017720385

LILLESAND, T. M.; KIEFER, R. W.; CHIPMAN, J. W. Remote sensing and image interpretation. In John Wiley e Sons, USA, 2015.

LISBOA, C. E. M. Considerações sobre o mapeamento do Brasil a curto prazo: Tentativa de uma solução. Revista Brasileira de Cartografia, vol. 2, n. 1, p. 41-47, 1971.

LONGLEY, P. A. Geographical Information Systems: will developments in urban remote sensing and GIS lead to "better" urban geography? Progress in Human Geography, vol. 26, n. 2, p. 231-239, 2002.

https://doi.org/10.1191/0309132502ph366pr

MARSHALL, S. Planning, Design and the Complexity of Cities. In Portugali, J.; H. Meyer; Stolk, E.; Tan, E. (eds.). Complexity Theories of Cities Have Come of Age. Berlin: Springer, p. 191-205, 2011. https://doi.org/10.1007/978-3-642-24544-2 11

MULLIGAN, G. F.; CRAMPTON, J. P. Population growth in the world's largest cities. Cities, vol. 22, n. 5, p. 365-380, 2005. https://doi.org/10.1016/j.cities.2005.07.001

OpenStreetMap. OSM (2020). Mapa colaborativo. Disponível em http://www.openstreetmap.org Acesso em: 15 de maio de 2020.

$\begin{array}{llllll}\text { Caminhos de Geografia } & \text { Uberlândia-MG } & \text { v. 22, n. } 82 & \text { ago./2021 } & \text { p. 28-40 } & \text { Página } 39\end{array}$


PAULA, K. A.; FARIA, T. C. A. A interiorização dos campi das Instituições Federais de Ensino Superior (IFES) e seus impactos no espaço urbano: um estudo de caso a partir da cidade de Viçosa-MG. Revista Brasileira de Gestão Urbana, vol. 12., 2020. https://doi.org/10.1590/2175-3369.012.e20190089

PERKINS, T. Speed and accuracy improvements in FLAASH atmospheric correction of hyperspectral imagery. Optical Engineering, vol. 51, n. 11, 2012. https://doi.org/10.1117/1.OE.51.11.111707

RAMIREZ, F. L. B; ANDERSON, L. O.; FORMAGGIO, A. R. Normalização radiométrica automatizada para geração de mosaicos de imagens RapidEye sobre paisagens amazônicas, através da transformação IRMAD. In Anais do XVII Simpósio Brasileiro de Sensoriamento Remoto, p. 7619-7622, João Pessoa, INPE, 2015.

ROSA, R. Análise Espacial em Geografia. Revista ANPEGE, vol. 7, n. 1, p. 275-289, 2011. https://doi.org/10.5418/RA2011.0701.0023

SANTOS, M. Manual de Geografia Urbana. Edusp: São Paulo, 2012.

VARNA, G.; ADAMS, D.; DOCHERTY, I. Development networks and urban growth in small cities. European Urban and Regional Studies, vol. 27, n. 1, p. 70-85, 2020.

https://doi.org/10.1177/0969776418802919

WOODCOCK, C. E.; STRAHLER, A. H. The factor of scale in remote sensing. Remote Sensing of Environment, vol. 21, n. 3, p. 311-332, 1987. https://doi.org/10.1016/0034-4257(87)90015-0

YANG, X. Urban Remote Sensing: Monitoring, Synthesis and Modeling in the Urban Environment. Wiley-Blackwell, USA, 2011. https://doi.org/10.1002/9780470979563

YUAN, J.; NIU, Z. Evaluation of atmospheric correction using flaash. In Proceedings of the International Workshop on Earth Observation and Remote Sensing Applications, EORSA, 2008.

Recebido em:24/06/2020

Aceito para publicação em:28/10/2020 